\title{
Reduced Order Model Compensator Control of Species Transport in a CVD Reactor
}

\author{
G.M. Kepler, H.T. Tran, H.T. Banks \\ Center For Research in Scientific Computation \\ North Carolina State University, Raleigh, NC 27695
}

Corresponding author:

H.T. Banks, Center For Research in Scientific Computation

Box 8205, NCSU, Raleigh NC 27695

Telephone, 919-515-3968; Fax, 919-515-8967; Email, htbanks@eos.ncsu.edu

\begin{abstract}
We propose the use of proper orthogonal decomposition (POD) techniques as a reduced basis method for computation of feedback controls and compensators in a high pressure chemical vapor deposition (HPCVD) reactor. In this paper, we present a proof-of-concept computational implementation of this method with a simplified growth example for III-V layers in which we implement Dirichlet boundary control of a dilute Group III reactant transported by convection and diffusion to an absorbing substrate with no reactions. We implement the model-based feedback control using a reduced order state estimator based on observations of the flux of reactant at the substrate center. This is precisely the type of measurements available with current sensing technology. We demonstrate that the reduced order state estimator or compensator system is capable of substantial control authority when applied to the full system. In principle, these ideas can be extended to more general HPCVD control situations by including multiple species with gas phase reactions and surface reactions.
\end{abstract}




\section{Introduction}

Production of advanced optoelectronic integrated circuits requires stringent control of layer thickness and composition. These requirements can be addressed, in part, through open-loop optimization $[6,21,34]$. However, because of process variability and the increasing demands put on control of layer thickness and composition by state-of-the art devices, real-time control of film growth is desirable [17, 37, 35, 7].

Many materials utilized in today's electronics industry are manufactured using chemical vapor deposition (CVD) processes operating at low pressure. However, there are also materials of potential industrial use that can not be adequately produced at desirable process temperatures under low pressure conditions, e.g., InN films that exhibit relatively high decomposition pressure as compared to other III-V compounds. In such cases, it may be desirable to extend the CVD processing to higher pressures. We are presently involved in a collaboration with material scientists at N.C. State University to design and build such a HPCVD reactor with real-time sensing and control as an innovative feature of this prototype reactor.

There are many technical issues associated with real-time control of a HPCVD reactor, such as coupling of species transport, gas phase reactions, and surface reactions to film growth and development of suitable sensing technology. We propose using accurate model simulations of species transport and reactions to provide data about the state of the system and to design state feedback controllers. In general, a full mathematical model describing transport processes in CVD systems is given by a system of nonlinear partial differential equations representing the continuity, momentum, energy, and species equations of state. Therefore, numerical simulations and control designs of such systems using finite element, finite difference, or spectral methods will lead to a very large system of ordinary differential equations rendering real-time full model-based feedback control design infeasible. To reduce the dimensionality of the system, we propose using the method of proper orthogonal decomposition (POD) to more efficiently represent the system data [27]. Recognizing that observation of the full state of the system can not be experimentally realized with current sensing technology, we propose using a state estimator or compensator based on P-polarized reflectance spectroscopy (PRS) sensing technology, which provides the capability for real-time observation of the growth rate [5].

In this paper, we present a proof-of-concept implementation of this method with a simplified growth example for III-V layers, which can, in general, be extended to more experimentally relevant cases. Normally, during the growth of III-V films the Group III reactants determine the growth rate and composition, since the Group V reactants are supplied in overabundance, while the Group III reactants, possessing high sticking coefficients, are supplied in dilute amounts relative to the carrier gas. We present im- 
plementation of Dirichlet boundary control of a dilute Group III reactant transported by convection and diffusion to an absorbing substrate with no reactions. Computational fluid dynamics (CFD) simulations provide data on the full system and are used to construct a POD reduced order model.

The control/compensator problem (or LQG tracking problem) is formulated as a linear quadratic regulator (LQR) tracking problem, where the state of the system is estimated via a compensator gain from observation of the flux of the reactant to the substrate (assumed proportional to the growth rate), with the observed flux attempting to track a desired flux value. Dirichlet boundary control at the inlet determines the mass fraction of the incoming Group III reactant. We demonstrate that the reduced order state estimator or compensator system is capable of substantial control authority when applied to the full system. In principle, these ideas can be extended to more general HPCVD control situations by including multiple species with gas phase reactions and surface reactions.

The utility of POD (also known under the names of principal component analysis [19] and Karhunen-Loève expansion [20, 24]) as a method of feature extraction is well known in statistical and pattern recognition fields [16] and has been applied in such diverse areas as materials processing [27] and characterization of human faces [30]. The POD method is a linear transformation of a multivariate data set into an optimal set of uncorrelated variables (POD modes). The original multivariate data can be written as linear combinations of the POD modes. In many cases the POD modes more efficiently describe the variability of the original data and some dimensional reduction is possible by retaining only the most important modes.

POD based approximation methods have been widely discussed in the context of turbulent coherent flows $[3,12,13,15,18,25,31]$ - see also the surveys [11, 26]. More recently, the possibility of POD based control design has been proposed. In particular, the first uses of POD approximations in optimization or open loop control were demonstrated in $[27,34]$ for model-based methods and subsequently in [28] for model-free methods. The first use of POD approximations in feedback control design was reported in [8, 9] for control of periodically disturbed structures. POD methods for state feedback were also used at approximately the same time in [22] in an example for Burgers' equation and were later used in [1] in control design for the heat equation. To our knowledge, the results reported below offer the first computational evidence of the successful use of POD based methods for feedback control and compensator design in tracking problems for a flow related system where only partial state observations are available: in this case in the control of pulsed metallic vapors in a CVD reactor.

There are a number of nontrivial issues related to the use of reduced basis methods in general and POD methods in particular as a foundation for approximation methods in 
infinite dimensional systems such as those modeled by distributed parameter or partial differential equation systems. The foremost revolves around whether the infinite dimensional system itself can be approximated well by a finite span of basis elements. That is, are the features of importance in a given investigation of the system essentially finite dimensional in nature? There is growing evidence that the answer to this question is positive for many structural systems and for a substantial number of fluid and electromagnetic applications. Even when this question can be answered in the affirmative, it is not at all clear that one can use such an approach for control design or in inverse problems related to damage detection (another area we are currently investigating with POD based methods).

In particular, if one uses 'snapshots' of the uncontrolled system (as was done in $[8,9]$ ) to construct the POD basis elements, there is little reason to expect that control design based on this finite dimensional approximation will be effective when applied to the original system. The controlled original system itself may require a different set of finite dimensional elements for efficient approximation (or, worse yet may not be amenable to a low order basis approximation). The results of $[8,9]$ suggest that, at least in some situations, happily this is not the case. The approach we offer in this paper (which was also successfully used in open loop control problems in [27]) illustrates that another tactic can be effective. That is, one may wish to 'snapshot' on the system under several levels of control input (not, in general, derived from any optimal or suboptimal design) in order to take snapshots on the system under nontrivial control inputs.

In Section 2 below we describe the particular system under investigation here and describe the simulations needed to develop POD based control design. A sample of some of our computational results based on this approach is given in Section 3. Brief conclusions are then summarized.

\section{Methods}

\subsection{Governing Equations}

The governing equations for the transport dynamics in CVD processes are described by the following system of partial differential equations: (mass)

$$
\frac{\partial \rho}{\partial t}+\vec{\nabla} \cdot(\rho \vec{v})=0,
$$


(momentum)

$$
\rho\left(\frac{\partial \vec{v}}{\partial t}+\vec{v} \cdot \vec{\nabla} \vec{v}\right)=-\vec{\nabla} P+\vec{\nabla} \cdot \vec{\tau}-\rho \vec{g}
$$

where the viscous stress tensor is of the form

$$
\vec{\tau}=-\frac{2}{3} \mu(\vec{\nabla} \cdot \vec{v}) \vec{I}+\mu\left(\vec{\nabla} \vec{v}+\vec{\nabla} \vec{v}^{T}\right),
$$

(energy)

$$
\rho c_{p}\left(\frac{\partial T}{\partial t}+\vec{v} \cdot \vec{\nabla} T\right)=\vec{\nabla} \cdot(k \vec{\nabla} T)
$$

and (species)

$$
\frac{\partial Y}{\partial t}+\vec{v} \cdot \vec{\nabla} Y=\frac{1}{\rho} \vec{\nabla} \cdot(\rho D \vec{\nabla} Y),
$$

where in (5) we assume that there are no reactions in the gas phase. In addition, $\vec{g}$ is the gravitational acceleration, $\vec{v}, T$, and $P$ are the velocity, temperature, and pressure, $\mu, c_{p}$, and $k$ are the viscosity, specific heat, and conductivity of the carrier gas, $D$ is the diffusivity of the reactant, and $Y$ is the mass fraction of the reactant. The density variations are modeled as $\rho=\rho_{0}\left[1-\beta\left(T-T_{0}\right)\right]$, where $T_{0}$ is a reference temperature, $\rho_{0}$ is a reference density calculated from the ideal gas law at the reference temperature and reactor pressure, and $\beta$ is the volume coefficient of expansion $(\beta=1 / T)$. The boundary conditions for the above system of equations (1)-(5) will be given in subsequent sections.

We summarize our use below of the system (1)-(5) in numerically investigating reduced order control and compensator design. Since only a trace amount of Group III reactant is mixed with the carrier gas, we can, first, solve (1)-(4) for steady state solutions using a commercially available CFD finite element software package. These values are then employed in a transient simulation of (5) to solve (again using standard finite element procedures) for values to be used as 'simulated experimental data' or 'time snapshots' for (5). We then carry out the POD procedure described below to produce POD basis elements $\left\{\Psi_{i}\right\}_{i=1}^{K}$ using POD nodal values $\left\{z_{k}^{N}\right\}_{k=1}^{K}$. The control problem for (5) is then approximated in penalty form (10), which is then written in weak or variational form (11). A standard Galerkin (quadratic) finite element method is used to approximate solutions of (11); this yields the full dimensional approximation of (11) that plays the role of a reactor simulator. A reduced order $(M<<K)$ POD basis is used to approximate solutions of (11) via a Galerkin procedure which yields the finite dimensional (low order) approximation of (11) to be used in LQG control/compensator gain design. 


\subsection{CFD simulations}

Specifically, we use a 2D rectangular geometry (Fig. 1) representing the longitudinal cross section through the center of an HPCVD reactor with dimensions (height $=0.011$ $\mathrm{m}$, length $=0.156 \mathrm{~m}$, substrate length $=0.048 \mathrm{~m}$ ) that are similar to the present N.C. State reactor design.

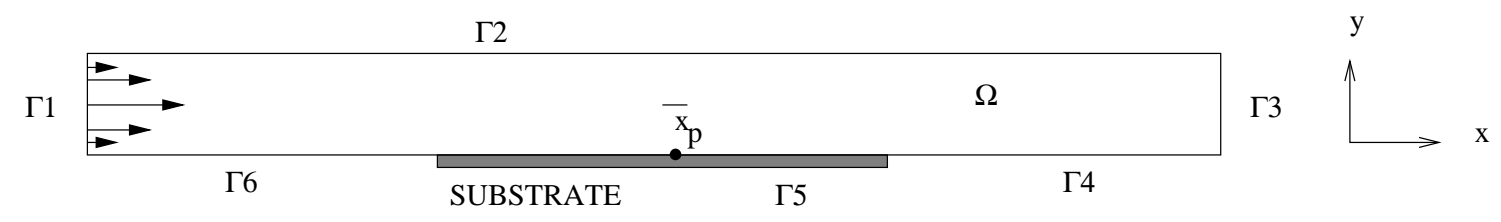

Figure 1: HPCVD reactor geometry.

The governing equations are discretized using the Galerkin finite element method with weighted residuals for the degrees of freedom $(\vec{v}, P$, and $T)$. A mixed formulation with 132 quadrilateral elements (corresponding to 453 nodes) is used with piecewise linear discontinuous elements for pressure, and quadratic (8-noded) elements for the other degrees of freedom. Solutions for the variables $\vec{v}, P, T$, and $Y$ are obtained using simulations with commercially available code (FIDAP, Fluid Dynamics International, Evanston, IL) on a Silicon Graphics Power Indigo 2.

Steady-state simulation. Under the dilute approximation, steady-state solutions for the velocity, temperature, and pressure are independent of the reactant concentration and are, of course, time-independent. Therefore, the spatially-dependent solutions for $\vec{v}$ and $T$, appearing in (5), are obtained from a steady-state simulation of (1)-(4) at atmospheric pressure with hydrogen carrier gas. Temperature dependent values for $\mu, k$, and $c_{p}$ necessary for solution of (1)-(4) are linearly interpolated from measurements taken from the available literature $[23,32,36]$. A parabolic velocity flow profile is specified at the inlet $(\Gamma 1)$, with an average inlet velocity of $0.1147 \mathrm{~m} / \mathrm{s}$. Room temperature boundary conditions are imposed at the inlet and along the upper wall (Г2). Along the bottom wall, the substrate $(\Gamma 5)$ temperature is fixed at $800^{\circ} \mathrm{K}$, with a non-linear temperature decrease from the substrate edge to the inlet $(\Gamma 6)$ and, similarly, from the substrate edge to the outlet $(\Gamma 4)$.

Reactant transport simulation. Trimethylindium is an organometallic precursor commonly used as a source material for CVD growth of III-V films where indium is one of the constituents (e.g., InN). A transient simulation of trimethylindium (TMI) convection and diffusion (5) is used to obtain data for construction of the POD modes. This system, 
including a nonzero boundary input, is given by

$$
\begin{aligned}
\frac{\partial Y}{\partial t}+\vec{v} \cdot \vec{\nabla} Y & =\frac{1}{\rho} \vec{\nabla} \cdot(\rho D \vec{\nabla} Y) & & \\
Y(0, \vec{x}) & =0 & & \\
Y(t, \vec{x}) & =1 & & \text { on } \Gamma 1 \\
Y(t, \vec{x}) & =0 & & \text { on } \Gamma 5 \\
\frac{\partial Y(t, \vec{x})}{\partial n} & =0 & & \text { on } \Gamma 2 \cup \Gamma 3 \cup \Gamma 4 \cup \Gamma 6,
\end{aligned}
$$

where $Y$ is the mass fraction of TMI and $\frac{\partial}{\partial n}$ denotes the outward normal derivative. Initially, the TMI mass fraction is assumed zero everywhere in the reactor. A normalized TMI mass fraction of one $(Y=1)$ is maintained as a nontrivial input 'control' at the inlet $(\Gamma 1)$. The reactor walls $(\Gamma 2, \Gamma 4$, and $\Gamma 6)$ are non-absorbing, and the substrate $(\Gamma 5)$ is assumed to be perfectly absorbing (concentration of zero). Values for $\vec{v}$ and $\rho$ appearing in (5) are provided by the steady-state solutions. Temperature dependent values for the diffusivity $D$ of TMI in hydrogen are linearly interpolated from values taken from the available literature [33]. Transport of the TMI is simulated using standard finite elements over a 2-s time period, sufficiently long for achieving a steady-state concentration in the reactor. Time integration is implemented using a backward Euler method with fixed time steps $(0.02 \mathrm{~s})$. Intermediate solution values are stored at each time step to be used later in construction of the POD basis elements.

\subsection{Construction of POD Modes}

The reactant transport simulation described above provides a multivariate data set consisting of $K$ vectors $X=\left\{x_{1}^{N}, x_{2}^{N}, \ldots, x_{K}^{N}\right\}$, each vector representing $N$ nodal values of the species concentration in the reactor at different times during the period that the reactant was entering the reactor and reaching its equilibrium distribution. In this calculation, 100 time snapshots are available but only $K=60$ are used in the POD construction (see Figure 3 and the associated discussion below). This original data set $X$ is transformed to a new set of uncorrelated variables (POD modes)

$$
Z=\left\{z_{1}^{N}, z_{2}^{N}, \ldots, z_{K}^{N}\right\}=X \Phi,
$$

where the columns of $\Phi=\left\{\phi_{1}^{K}, \phi_{2}^{K}, \ldots, \phi_{K}^{K}\right\}$ are the eigenvectors of the product matrix $\left(X^{\prime} X\right) \phi_{i}^{K}=\lambda_{i} \phi_{i}^{K}$, ranked, in descending order, with respect to the associated eigenvalue. The prime superscript denotes the transpose of the matrix. The POD modes $Z$ are orthogonal $z_{i}^{N} \cdot z_{j}^{N}=\lambda_{i} \delta_{i, j}$, and the transformation of variables preserves the data variability

$$
\sum_{k}^{K}\left(X^{\prime} X\right)_{k k}=\sum_{k}^{K}\left(Z^{\prime} Z\right)_{k k}=\sum_{k}^{K} \lambda_{k} .
$$


Expansion of the original data $X$ in terms of the most significant POD modes minimizes the mean square error of a reduced basis representation [16]

$$
X_{i j}^{M}=\sum_{k=1}^{M} Z_{i k} \Phi_{k j}^{\prime},
$$

where $M<K$. The most significant POD modes are those corresponding to the largest eigenvalues, since the ratio of an eigenvalue to the summation of eigenvalues, $\lambda_{k} / \sum_{j}^{K} \lambda_{j}$, gives the percentage of the mean square error unaccounted for by eliminating the corresponding POD mode $z_{k}^{N}$ [16] in the reduced basis representation. The best stopping point in the expansion (9) depends, in general, upon the application and various algorithms have been proposed [19]. For our feedback control/compensator application, we choose the order $M$ of our reduced system to be the maximum order such that the associated finite dimensional control system is both controllable and observable while an acceptable level (99.72\% in this case, see Section 3 below) of data variability is captured.

\subsection{Penalty Boundary Formulation of the Control Problem}

We use a penalty boundary formulation of the time-dependent species equation (5) without reactions to describe the transport of TMI in the reactor

$$
\begin{aligned}
\frac{\partial Y}{\partial t}+\vec{v} \cdot \vec{\nabla} Y & =\frac{1}{\rho} \vec{\nabla} \cdot(\rho D \vec{\nabla} Y) \\
Y(0, \vec{x}) & =y_{0}(\vec{x}) \\
\frac{\partial Y(t, \vec{x})}{\partial n} & =\frac{1}{\epsilon}(Y(t, \vec{x})-u(t)) \quad \text { on } \Gamma 1 \\
\frac{\partial Y(t, \vec{x})}{\partial n} & =\frac{1}{\epsilon} Y(t, \vec{x}) \quad \text { on } \Gamma 5 \\
\frac{\partial Y(t, \vec{x})}{\partial n} & =0 \text { on } \Gamma 2 \cup \Gamma 3 \cup \Gamma 4 \cup \Gamma 6,
\end{aligned}
$$

where $Y$ is the mass fraction of TMI, $\frac{\partial}{\partial n}$ denotes the outward normal derivative, and $u(t)$ is the control. Under sufficient regularity, one can argue that solutions of $(10)$, in the limit as $\epsilon \rightarrow 0$, approximate solutions for the problem with conditions $Y(t, \vec{x})=u(t)$ on $\Gamma 1$, and $Y(t, \vec{x})=0$ at $\Gamma 5$ (see $[4,10]$ for related discussions). For the results presented here, a value of $\epsilon=1 \times 10^{-3}$ is used.

Writing (10) in weak form with test functions $w_{j}$, we obtain

$$
\begin{aligned}
\int_{\Omega} \frac{\partial Y}{\partial t} w_{j} d \Omega= & -\int_{\Omega}(\vec{v} \cdot \vec{\nabla} Y) w_{j} d \Omega-\int_{\Omega} D \vec{\nabla} Y \cdot \vec{\nabla} w_{j} d \Omega+\int_{\Omega} \frac{1}{\rho} w_{j} D \vec{\nabla} Y \cdot \vec{\nabla} \rho d \Omega \\
& +\frac{1}{\epsilon} \int_{\Gamma 1, \Gamma 5} w_{j} D Y d s-\frac{1}{\epsilon} \int_{\Gamma 1} w_{j} D u d s
\end{aligned}
$$


Spatially dependent values for $\vec{v}, T, \rho$, and $D$ are interpolated from the nodal values obtained from the CFD simulations.

As we have explained above, two discretization formulations are applied to (11). The first formulation, a finite element approximation, produces the full system (which plays the role of a reactor simulator). In this case, the TMI concentration in (11) is approximated using standard finite element discretization with $N=453$ quadratic interpolation functions $\psi_{i}$ and $N$ nodal coefficients $y_{i}^{N}$

$$
Y^{N}(t, \vec{x})=\sum_{i=1}^{N} y_{i}^{N}(t) \psi_{i}(\vec{x}) .
$$

Choosing the test functions to be $w_{j}=\psi_{j} j=1,2, \ldots, N$, we obtain in a standard way the matrix equation

$$
\dot{y}^{N}(t)=A^{N} y^{N}(t)+B^{N} u(t),
$$

where $A^{N}$ is an $N \times N$ matrix, $B^{N}$ is a vector of length $N$, and the control $u$ is a scalar control function.

The second discretization formulation produces the reduced basis model. In this case, we first use the POD modes $\left\{z_{k}^{N}\right\}$ to obtain the POD elements

$$
\Psi_{k}(\vec{x})=\sum_{i=1}^{N} z_{k i} \psi_{i}(\vec{x}), \quad k=1,2, \ldots, K,
$$

where the functions $\psi_{i}(\vec{x})$ are the finite element quadratic interpolation functions. The reactant mass fraction is approximated as a linear combination of the POD basis elements corresponding to the most significant POD modes

$$
Y^{M}(t, \vec{x})=\sum_{i=1}^{M} y_{i}^{M}(t) \Psi_{i}(\vec{x})
$$

where, in this case, $M<<K<<N$. Application of this approximation to (11) (in this case we use POD test functions $\left.w_{i}=\Psi_{i} i=1,2, \ldots, M\right)$ yields

$$
\dot{y}^{M}(t)=A^{M} y^{M}(t)+B^{M} u(t),
$$

where $A^{M}$ is an $M \times M$ matrix, and $B^{M}$ is a vector of length $M$. 


\subsection{Control}

To control the reduced order system (16), we observe the flux of reactants $-\rho D \frac{\partial Y}{\partial n}$ to the center of the substrate $\left(\vec{x}_{p}\right)$, which is approximated as

$$
q^{M}=-\left.\sum_{k=1}^{M} \rho D \frac{\partial \Psi_{k}}{\partial n}\right|_{\vec{x}_{p}} y_{k}(t)=\left(H^{M}\right)^{\prime} y^{M}(t) .
$$

We seek the optimal control $u^{*}$ for (16) such that the output $q^{M}$ tracks a signal $q_{T}$ (the desired flux at $\left.\vec{x}_{p}\right)$, minimizing the generalized performance index (e.g., see $\left.[2,29]\right)$

$$
V\left(y_{0}, u(\cdot)\right)=\int_{0}^{\infty}\left[u^{\prime} R u+\left(\bar{q}^{M}\right)^{\prime} Q_{2} \bar{q}^{M}+\left(q^{M}-q_{T}\right)^{\prime} Q_{1}\left(q^{M}-q_{T}\right)\right] d t .
$$

Choosing $Q_{2}=r_{2} I, \quad R=I, \quad Q_{1}=r_{1}, \quad \bar{q}^{M}=\left(\bar{H}^{M}\right)^{\prime} y^{M}, \quad\left(\bar{H}^{M}\right)^{\prime}=I-L^{M}\left(H^{M}\right)^{\prime}$, and $L^{M}=H^{M}\left(\left(H^{M}\right)^{\prime} H^{M}\right)^{-1}$, we can rewrite this performance index as

$$
V\left(y_{0}, u(\cdot)\right)=\int_{0}^{\infty}\left[u^{\prime} R u+\left(y^{M}-y_{T}^{M}\right)^{\prime} Q\left(y^{M}-y_{T}^{M}\right)\right] d t
$$

where $r_{1}$ and $r_{2}$ are design parameters, $I$ is the identity matrix, $Q=\overline{H^{M}} Q_{2}\left(\overline{H^{M}}\right)^{\prime}+$ $H^{M} Q_{1}\left(H^{M}\right)^{\prime}$, and $y_{T}^{M}=L^{M} q_{T}$ is the desired state trajectory. This is a standard formulation for a 'tracking' control problem $[2,29]$ and for which a complete theory is known (for a summary and references see Chapter 7 of [7]). The optimal control is given by $u^{*}=-K^{M} y^{M}-g^{M}$, where the gain is given by (see p84-85 of [2], [29], or [7])

$$
K^{M}=R^{-1}\left(B^{M}\right)^{\prime} \Pi
$$

$\Pi$ satisfies the algebraic Riccati equation (ARE)

$$
0=\Pi A^{M}+\left(A^{M}\right)^{\prime} \Pi-\Pi B^{M} R^{-1}\left(B^{M}\right)^{\prime} \Pi+Q,
$$

and the tracking term $g^{M}$ is given by

$$
g^{M}=R^{-1}\left(B^{M}\right)^{\prime} b
$$

where

$$
b=\left[\left(A^{M}-B^{M} K^{M}\right)^{\prime}\right]^{-1} Q y_{T}^{M} .
$$

\subsection{State Estimation in the Reduced Order Model}

Application of the tracking control to the reduced order model (16) yields

$$
\begin{aligned}
\dot{y}^{M} & =A^{M} y^{M}-B^{M} K^{M} y^{M}-B^{M} g^{M} \\
q^{M}(t) & =\left(H^{M}\right)^{\prime} y^{M}(t)
\end{aligned}
$$


with $K^{M}$ and $g^{M}$ defined in (20) and (22), respectively. Note that this formulation requires complete reduced order state feedback for the gain $K^{M}$ of (20). Since the full state of the system (or even the reduced order system) in the reactor can not be observed, we implement a state estimator or compensator design based on observation (17) of the flux at the center of the substrate. This yields (see [29] or Chapter 8 of [7] and the references therein) the coupled system

$$
\begin{aligned}
\dot{y}^{M} & =A^{M} y^{M}-B^{M} K^{M} y_{e}^{M}-B^{M} g^{M} \\
\dot{y}_{e}{ }^{M} & =A_{c} y_{e}^{M}+F^{M}\left(H^{M}\right)^{\prime} y^{M}-B^{M} g^{M},
\end{aligned}
$$

where $y_{e}^{M}$ is the $M \times 1$ vector approximation to the approximate state $y^{M}$, the compensator system operator $A_{c}$ is given by

$$
A_{c}=A^{M}-F^{M}\left(H^{M}\right)^{\prime}-B^{M} K^{M}
$$

and the compensator gain (Kalman filter) is given by

$$
F^{M}=\Sigma H^{M} V^{-1} \text {. }
$$

The matrix $\Sigma$ satisfies the dual ARE given by

$$
A^{M} \Sigma+\Sigma\left(A^{M}\right)^{\prime}-\Sigma H^{M} V^{-1}\left(H^{M}\right)^{\prime} \Sigma+U=0
$$

where $U$ is a symmetric positive semi-definite matrix design parameter and $V$ is a symmetric positive definite matrix design parameter. We choose $U=I$ and $V=r_{3}$, where $r_{3}$ is a third parameter at our disposal in designing the overall feedback control/compensator system.

This implementation of the state estimator yields the following closed-loop system for 'optimal' control of the reduced order model

$$
\left(\begin{array}{c}
\dot{y}^{M} \\
\dot{y}_{e}^{M}
\end{array}\right)=\left(\begin{array}{cc}
A^{M} & -B^{M} K^{M} \\
F^{M}\left(H^{M}\right)^{\prime} & A^{M}-B^{M} K^{M}-F^{M}\left(H^{M}\right)^{\prime}
\end{array}\right)\left(\begin{array}{c}
y^{M} \\
y_{e}^{M}
\end{array}\right)+\left(\begin{array}{c}
-B^{M} g^{M} \\
-B^{M} g^{M}
\end{array}\right)
$$

with the optimal control

$$
u^{*}=-K^{M} y_{e}^{M}-g^{M} .
$$

\subsection{Control of the Full System Using a Reduced Order State Estimator}

Use of the design given in (29) and (30) can be expected to produce a stabilized and generally efficient system for control of the reduced order model (16). However, this is 
not the issue of practical importance in our efforts. The goal, of course, is to design a feedback control/compensator system for (10) (or in weak form (11)), which we hope will be a good approximation (for proper choice of $\epsilon$ ) for the actual physical dynamics (1)-(5), i.e., transient solutions of (5) with (1)-(4) in steady state. Thus, the real measure of the value of the control/compensator system of Sections 2.5-2.6 is how well it performs when used in the actual physical system. Short of applying the control/compensator system to the physical reactor in experiments, our best assessment of its utility is when applied to the 'full' system (13). That is, we should computationally test the reduced order control/compensator design based on (20)-(23), (26)-(30) in the system (13).

Application of the reduced order tracking control to the full system with the reduced order state estimator yields

$$
\left(\begin{array}{l}
\dot{y}^{N} \\
\dot{y}_{e}^{M}
\end{array}\right)=\left(\begin{array}{cc}
A^{N} & -B^{N} K^{M} \\
F^{M}\left(H^{N}\right)^{\prime} & A^{M}-B^{M} K^{M}-F^{M}\left(H^{M}\right)^{\prime}
\end{array}\right)\left(\begin{array}{c}
y^{N} \\
y_{e}^{M}
\end{array}\right)+\left(\begin{array}{c}
-B^{N} g^{M} \\
-B^{M} g^{M}
\end{array}\right)
$$

where $H^{N}$ is the observation vector for the full-dimensional system $q^{N}=\left(H^{N}\right)^{\prime} y^{N}(t)$, and $H^{M}, K^{M}, g^{M}$, and $F^{M}$ are as defined in (17), (20), (22), and (27), respectively. The optimal control is given by (30). We report on our simulations for system (31) with different values of the design parameter $r_{1}$ in the results of Section 3. Values of $r_{2}$ and $r_{3}$ were varied and then fixed at nominal performance values in the calculations reported below.

\subsection{Control Implementation}

The governing equations (10) are nondimensionalized prior to the calculation of the coefficient matrices using a length scale of $1 \times 10^{-2} \mathrm{~m}$, a diffusion coefficient scale of $1.15 \times 10^{-3}$ $\mathrm{m}^{2} / \mathrm{s}$, and a density scale of $4.04 \times 10^{-2} \mathrm{~kg} / \mathrm{m}^{3}$. The POD modes and the coefficient matrices $A^{N}, B^{N}, H^{N}, A^{M}, B^{M}$, and $H^{M}$ in (31) are calculated using in-house C programs. All other matrix calculations are implemented in the Matlab computing environment (The Math Works Inc., Natick, MA). The optimal gain matrix $K^{M}$ and Riccati solution $\Pi$ are determined using Matlab's $\operatorname{lq}()$ function and the dynamical equations are integrated using Matlab's ode23s() solver. 


\section{Results}

We report in sequential form results from the series of computations and simulations described in detail in Section 2.

\subsection{CFD Simulation Results}

We first report on results of the CFD package simulations described in Section 2.2. Contour plots of the steady-state solutions of the temperature and the x-component of the velocity (Fig. 2a-b) give an indication of the transport conditions in the reactor. With the top wall and inlet maintained at room temperature, there is a steep temperature gradient (Fig. 2a) upstream from the substrate. In the region above the substrate, the isotherms run parallel to the hot substrate and the opposite cold wall. The velocity of the gas increases more than 4-fold as it passes in the vicinity of the hot substrate (Fig. $2 \mathrm{~b}$ ), while the $\mathrm{y}$-component of the velocity (not shown) remains small throughout the reactor.

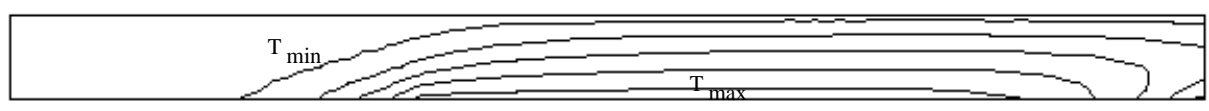

(a) Temperature

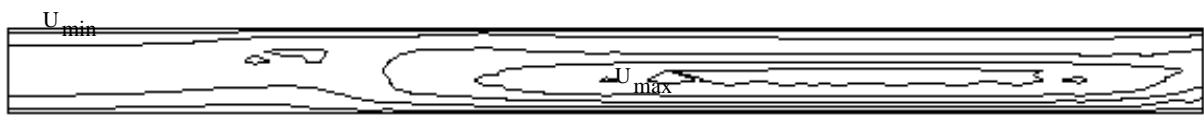

(b) x-component of velocity

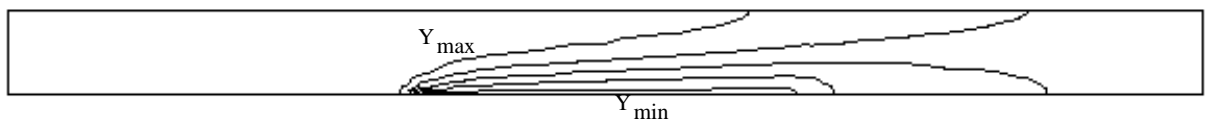

(c) TMI concentration

Figure 2: Contour plot of steady-state values for the (a) temperature in $100^{\circ} \mathrm{K}$ steps with $\mathrm{T}_{\text {max }}=750^{\circ} \mathrm{K}$ and $\mathrm{T}_{\min }=350^{\circ} \mathrm{K}$, (b) $\mathrm{x}$-component of the velocity in $0.1 \mathrm{~m} / \mathrm{s}$ steps with $\mathrm{v}_{\max }=0.45$ and $\mathrm{v}_{\min }=0.05$ $\mathrm{m} / \mathrm{s}$, and the (c) equilibrium distribution of TMI $2 \mathrm{~s}$ after the reactants entered the reactor, with contour lines representing steps of 0.2 in the normalized TMI mass fraction $\left(\mathrm{Y}_{\max }=0.9\right.$ and $\left.\mathrm{Y}_{\min }=0.1\right)$. 
A contour plot of the equilibrium distribution of TMI (at $t=2 \mathrm{~s}$ ) shows a steep gradient in the TMI concentration (Fig. 2c) at the leading edge of the substrate. There is a corresponding peak in the flux of TMI to the upstream end of the substrate (not shown). A plot of the average flux of TMI to the substrate as a function of time (Fig. 3) shows that an equilibrium value was reached approximately $1 \mathrm{~s}$ after the reactant was introduced into the reactor.

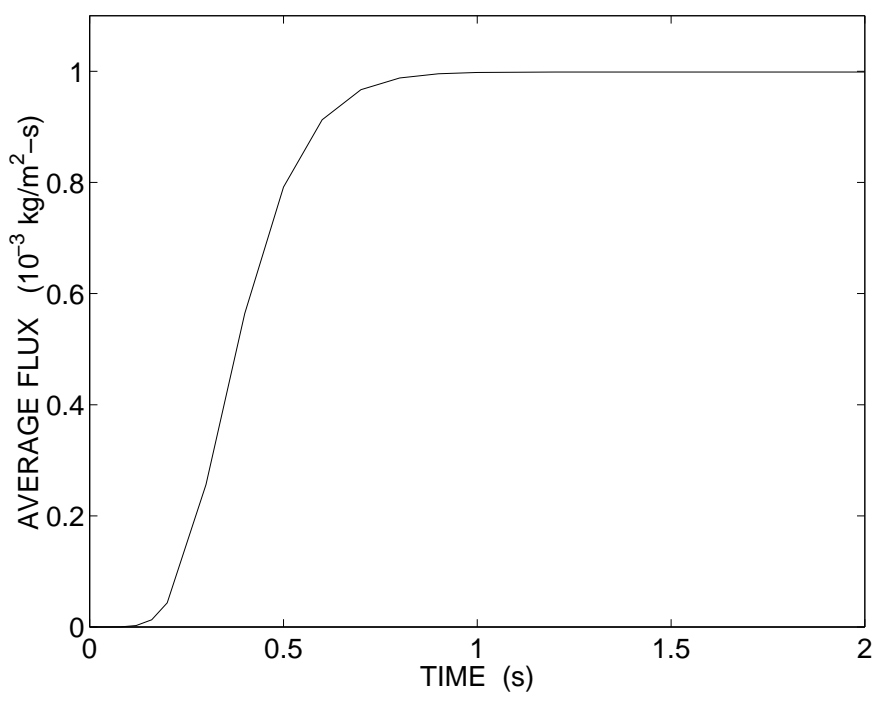

Figure 3: Average flux of TMI to the substrate as a function of time. 


\subsection{Construction of POD Modes}

Since equilibrium is reached after one second (Fig. 3), only the first sixty intermediate solution vectors $(K=60)$, representing the transport of TMI into the reactor from 0 $1.2 \mathrm{~s}$, are used to construct the POD modes. Each solution vector represents the TMI concentration at the 453 nodal points and corresponds to a time increment of 0.02-s in the time range from 0 to 1.2 seconds. A plot of the captured variability $\left(\sum_{j=1}^{M} \lambda_{j} / \sum_{j}^{K} \lambda_{j}\right)$ as a function of the number of modes $(M \leq 60)$ used (Fig. 4) shows that the original data is well-represented by 7 modes, or fewer. This strongly suggests using $M \leq 7$ in our reduced order model.

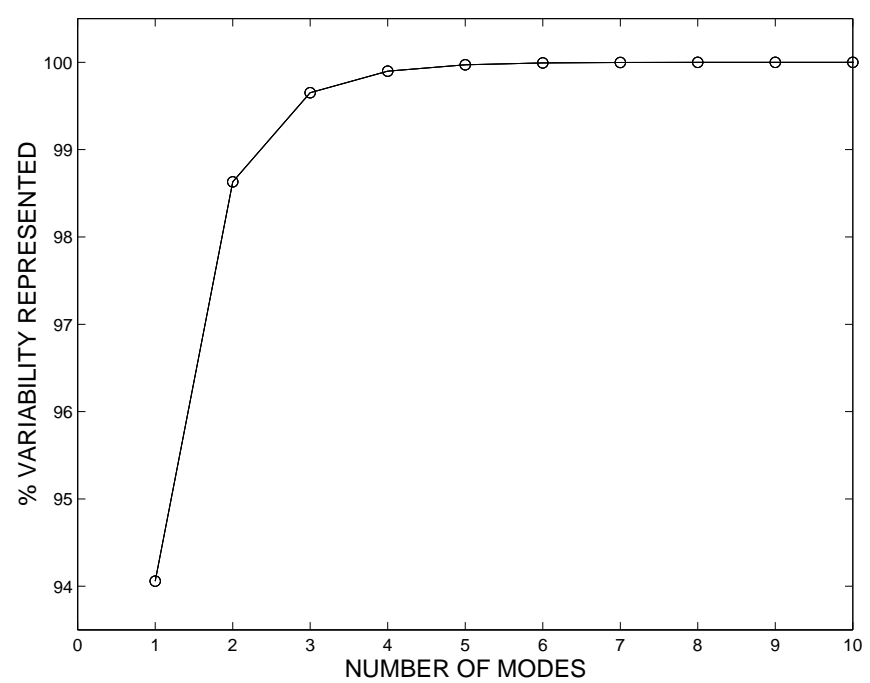

Figure 4: Total percent variability captured as a function of the number of modes.

The rank of the controllability matrix has been found to be a useful criterion (see the discussion in $[8,9]$ ) for determining the number of modes to use in control design applications for the reduced basis representation (15). The controllability of the linear system (16) is determined from the rank of the controllability matrix $\mathcal{C}$, where

$$
\mathcal{C}\left(\mathrm{A}^{\mathrm{M}}, \mathrm{B}^{\mathrm{M}}\right)=\left[\mathrm{B}^{\mathrm{M}}\left|\mathrm{A}^{\mathrm{M}} \mathrm{B}^{\mathrm{M}}\right|\left(\mathrm{A}^{\mathrm{M}}\right)^{2} \mathrm{~B}^{\mathrm{M}}|\ldots|\left(\mathrm{A}^{\mathrm{M}}\right)^{\mathrm{M}-1} \mathrm{~B}^{\mathrm{M}}\right] .
$$

Using standard results from optimal control theory [14], the $M$-dimensional linear system (16) is controllable if, and only if, the rank of the controllability matrix is equal to $M$. Similarly, the rank of the observability matrix $\mathcal{O}$ indicates the observability of the linear system (24), where

$$
\mathcal{O}\left(\mathrm{A}^{\mathrm{M}}, \mathrm{H}^{\mathrm{M}}\right)=\left[\mathrm{H}^{\mathrm{M}}\left|\left(\mathrm{A}^{\mathrm{M}}\right)^{\prime} \mathrm{H}^{\mathrm{M}}\right|\left(\left(\mathrm{A}^{\mathrm{M}}\right)^{2}\right)^{\prime} \mathrm{H}^{\mathrm{M}}|\ldots|\left(\left(\mathrm{A}^{\mathrm{M}}\right)^{\mathrm{M}-1}\right)^{\prime} \mathrm{H}^{\mathrm{M}}\right] .
$$


For our system under investigation here, the ranks of the controllability and observability matrices are recorded in Table 1 as a function of the number of modes in the reduced order model. The Table demonstrates that the rank of the controllability does not increase with additional modes after addition of the fifth mode. Similarly, the rank of the observability matrix does not increase with additional modes after addition of the sixth mode. Based on these results, we use in our control/compensator applications the first five most significant POD modes $(M=5)$, capturing $99.72 \%$ of the data variability, to construct our reduced order model (16).

Table 1: Rank of the Controllability and Observability Matrices

\begin{tabular}{ccc}
\hline Reduce Order Model & \multicolumn{2}{c}{ Rank } \\
Dimension M & $\mathcal{C}$ & $\mathcal{O}$ \\
\hline 1 & 1 & 1 \\
2 & 2 & 2 \\
3 & 3 & 3 \\
4 & 4 & 4 \\
5 & 5 & 5 \\
6 & 5 & 6 \\
7 & 5 & 6 \\
\hline
\end{tabular}




\subsection{Control of Full System Using the Reduced Order State Es- timator}

We carried out numerous simulations in investigating performance of the control/compensator design. A set of representative results are depicted here, where the reduced order state estimator is used to control the full system (31) using a non-dimensional tracking value of $q_{T}=0.183$ and design parameter values of $r_{1}=100,180$, or $10000, r_{2}=1 \times 10^{-5}$, and $r_{3}=1 \times 10^{4}$. Initially, the solution vector for the estimated state is given a small nonzero value $\left(y_{e}^{M}=1 \times 10^{-4}\right)$, while the full system solution is given an initial value of zero $\left(y^{N}=0\right)$. As a result of the latter condition, the observed flux to the substrate (Fig. 5) is initially zero and this zero flux persists for the time it takes the reactant introduced at the inlet to reach the substrate. (Recall that the control value represents the reactant mass fraction at the inlet.) As time progresses, the observed flux approaches and oscillates about the target flux value. The amplitude of the oscillations increases as the control parameter $r_{1}$ is increased, i.e., as more weight is placed on achieving the target flux. Eventually a steady-state flux value is attained, which exceeds the target flux value for the case of $r_{1}=10000$ and undershoots the target flux value for the case of $r_{1}=100$. With a design parameter value of $r_{1}=180$, the observed flux achieves the target flux value. The steady state flux attained with the control parameter value $r_{1}=10000$ is essentially the asymptotic value for large $r_{1}$, i.e., there is essentially no difference between the steady state results for $r_{1}=1000$ and $r_{1}=10000$.

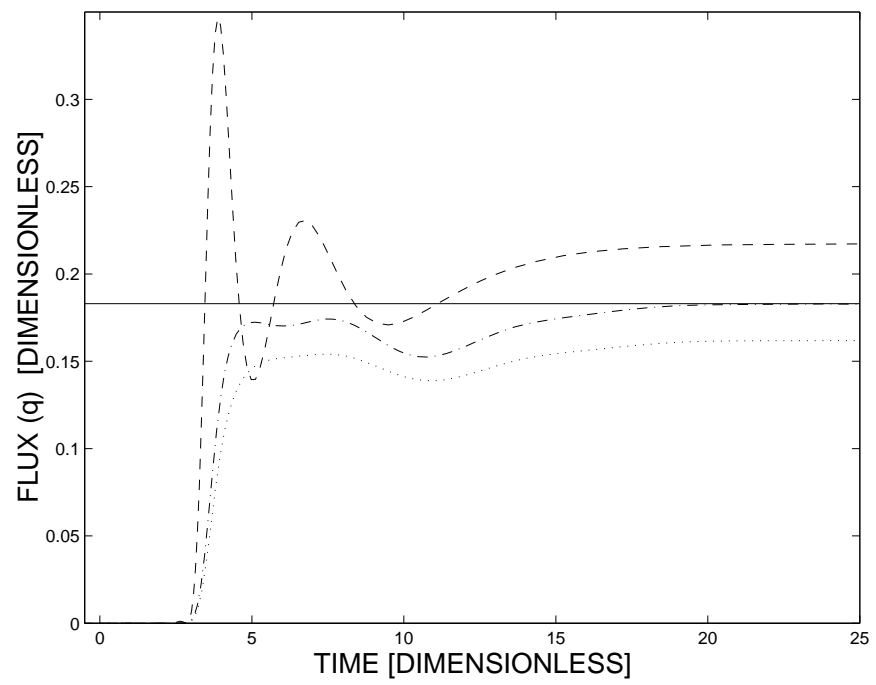

Figure 5: Observed flux compared to the desired flux (solid line) as a function of non-dimensional time with different levels of control: $r_{1}=100$ (dotted line), $r_{1}=180$ (dash-dot line), $r_{1}=10000$ (dashed line). 
A plot the norm of the difference between the full system solution and the state estimated solution as a function of time (Fig. 6) also shows oscillations, as initially, the state estimated solution did not closely track the full system solution. (Recall that the compensator or state estimator is an asymptotic estimator - see $[2,7])$. The greater the level of control (larger $r_{1}$ ) the larger are the magnitude of the oscillations. The oscillations, representing overshooting and undershooting of the adjustments to the state estimated solution, eventually (asymptotically) are attenuated and the difference between the two solutions reaches a steady-state value.

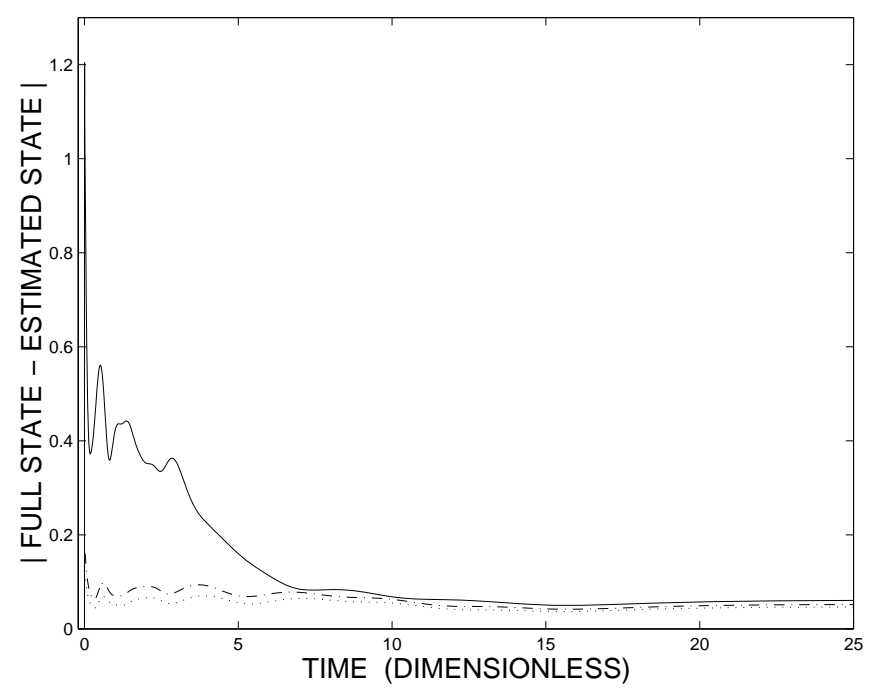

Figure 6: The norm of the difference between the full state and the POD estimated state with different levels of control: $r_{1}=100$ (dotted line), $r_{1}=180$ (dash-dot line), $r_{1}=10000$ (dashed line).

A plot of the control value $u$ as a function of time (Fig. 7) shows large initial control values oscillating and decreasing in time, eventually reaching steady-state values. Both the initial values and the magnitude of the oscillations increase as $r_{1}$ increases, as more weight is placed on achieving the desired flux and less weight is placed on the cost of control. Physically, the control values represent the mass fraction of TMI at the inlet. 


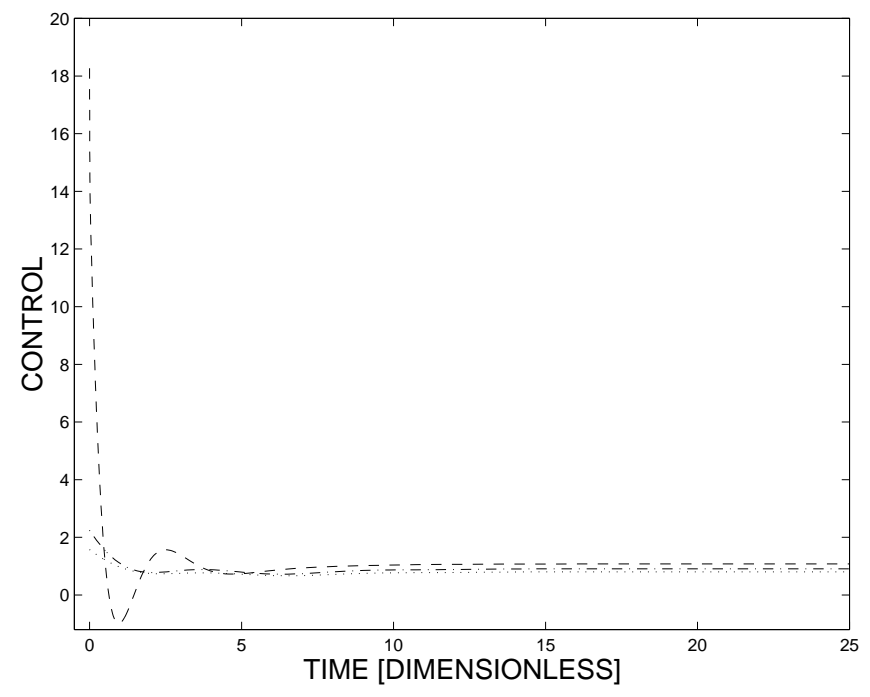

Figure 7: The control as a function of time with different levels of control: $r_{1}=100$ (dotted line), $r_{1}=180$ (dash-dot line), $r_{1}=10000$ (dashed line).

\section{Conclusion}

We have demonstrated a proof-of-concept computational implementation of reduced order feedback control of HPCVD III-V film growth which can, in principle, be extended to more experimentally relevant cases involving multiple species with reactions. We implement the control using a reduced order state estimator based on observations of the flux of TMI at the substrate center, which is precisely the type of measurements available with current PRS sensing technology. The POD-based design method allows us to reduce the order of the system by a factor of 90 with respect to a standard finite element representation (from $N=453$ to $M=5$ ), thus making real-time feedback control with partial state observations a feasible goal in HPCVD reactors operating in steady state flow regimes with pulsed vapor reactant inputs. This is, in fact, a conservative estimate of the dimensional reduction capability, since the number of nodes used here was minimized to reduce the simulation time of the full system.

\section{Acknowledgments}

This work was supported in part by DOD/AFSOR MURI Grant No. F49620-95-1-0447. The authors are grateful to Dr. Klaus Bachmann and other members of the applied math/material sciences team at N.C. State for numerous helpful conversations during our control methodology development efforts, part of which are reported here. 


\section{References}

[1] Atwell, J.A., and B. King, Proper orthogonal decomposition for reduced basis feedback controllers for parabolic equations, January, 1999, preprint.

[2] Anderson, B.D.O., and J.B. Moore, Optimal Control: Linear Quadratic Methods, (Prentice Hall, Englewood Cliffs, NJ, 1990).

[3] Aubry, N., P. Holmes, J.L. Lumley, and E. Stone, The dynamics of coherent structures in the wall region of a turbulent boundary layer, Journal of Fluid Mechanics 192, 115-173 (1988).

[4] Babuška, I., The finite element method with penalty, Mathematics of Computation 27, 221-228 (1973).

[5] Bachmann, K.J., N. Sukidi, C. Höpfner, C.Harris, N.A. Dietz, H.T. Tran, S. Beeler, K. Ito, and H.T. Banks. Real-time monitoring of steady-state pulsed chemical beam epitaxy by p-polarized reflectance, J. Crystal Growth 183, 323-337 (1998).

[6] Bachmann, K.J., H.T. Banks, C. Höpfner, G.M. Kepler, S.LeSure, S.D. McCall, and J.S. Scroggs, Optimal design of a high pressure organometallic chemical vapor deposition reactor, Mathematical and Computer Modelling (to appear).

[7] Banks, H.T., R.C. Smith, and Y. Wang, Smart Material Structures: Modeling, Estimation and Control, (Maisson/J.Wiley, Paris/Chichester, 1996).

[8] Banks, H.T., R.C.H. del Rosario, and R.C. Smith, Reduced order model feedback control design: Numerical implementation in a thin shell model, CRSC Tech Rep 98-27, N.C. State University, July 1998; IEEE Trans. Auto. Control, submitted.

[9] Banks, H.T., R.C.H. del Rosario, and R.C. Smith, Reduced order model feedback control design: Computational studies for thin cylindrical shells, CRSC Tech Rep 98-25, N.C. State University, June 1998.

[10] Barrett, J.W. and C.M. Elliot, Finite element approximation of the Dirichlet problem using the boundary penalty method, Numerische Mathematik 49, 343-366 (1986).

[11] G. Berkooz, Observations on the proper orthogonal decomposition, Studies in Turbulence, eds.: T.B. Gatski, S. Sarkar, and C.G. Speziale, (Springer-Verlag, New York, 1992), 229-247.

[12] Berkooz, G., P. Holmes, and J.L. Lumley, The proper orthogonal decomposition in the analysis of turbulent flows, Annual Review of Fluids Mechanics 25, N5:539-575, (1993). 
[13] Berkooz, G., P. Holmes J.L. Lumley, and J.C. Mattingly, Low-dimensional models of coherent structures in turbulence, Physics Reports-Review Section of Physics Letters 287, N4:338-384 (1997).

[14] Brogan, W.L., Modern Control Theory, (Prentice Hall, NJ, 1991).

[15] Chambers, D.H., R.J. Adrian, P. Moin, D.S. Stewart, and H.J. Sung, KarhunenLoève expansion of Burgers' model of turbulence, Phys. Fluids 31, 2573-2582 (1988).

[16] Fukunaga, K., Introduction to Statistical Pattern Recognition, (Academic Press, NY, 1972).

[17] Gevelber, M., M. Toledo-Quiñones, and M. Bufano, Towards closed-loop control of CVD coating microstructures, Materials Science \& Engineering A 209, 377-383 (1996).

[18] Iollo, A., S. Lanteri, and J.A. Désidéri, Stability properties of POD-Galerkin approximations for the compressible Navier-Stokes equations, INRIA Rep. de Rech. no. 3589, December, 1998, Sophia Antipolis.

[19] Jackson, J.E., A User's Guide to Principal Components, (Wiley, NY, 1991).

[20] Karhunen, K., Zur spektral theorie stochasticher prozesse, Ann. Acad. Sci. Fennicae Ser. A1 Math Phys. 37, (1946).

[21] Kepler, G.M, C. Höpfner, J.S. Scroggs, K.J. Bachmann, Feasibility of a vertical reactor for high pressure MOCVD, Materials Science \& Engineering B 57, 9-17 (1998).

[22] Kunisch, K., and S. Volkwein, Control of Burgers' equation by a reduced order approach using proper orthogonal decomposition, Optimierung und Kontrolle Bericht Nr. 138, September, 1998, Universitat Graz, Austria.

[23] Lide, D.R, and H.V. Kehiaian, CRC Handbook of Thermophysical and Thermochemical Data, (CRC Press, Boca Raton, 1994).

[24] Loève, M., Functions aleatoire de second ordre, Compte Rend. Acad. Sci. (Paris), 220 (1945).

[25] Lumley,, J.L., The structure of inhomogeneous turbulent flows, in Atmospheric Turbulence and Radio Wave Propagation, A.M. Yaglom and V.I. Tatarski, eds., (Nauka, Moscow, 1967) 166-178.

[26] Lumley,, J.L., Stochastic Tools in Turbulence, (Academic Press, New York, 1970). 
[27] Ly, H.V., and H.T. Tran, Proper orthogonal decomposition for flow calculations and optimal control in a horizontal CVD reactor, CRSC Tech Rep 98-13, N.C. State University, March 1998; Quarterly of Applied Mathematics, to appear.

[28] Ly, H.V, and H.T. Tran, Modeling and control of physical processes using proper orthogonal decomposition, Computers and Mathematics with Applications, to appear.

[29] Russell, D.L., Mathematics of Finite-Dimensional Control Systems: Theory and Design, (Marcel Dekker, New York, 1979).

[30] Sirovich, L., and M. Kirby, Low-dimensional procedure for the characterization of human faces, J. Opt. Soc. Am. 4, 519-524 (1987).

[31] Sirovich, L., Chaotic dynamics of coherent structures, Physica D 37, 126-145 (1989).

[32] Svehla, R.A., NASA Technical Report R-132, 1962.

[33] Theodoropolous, C., N.K. Ingle, T.J. Mountziaris, Z.-Y. Chen, P.L. Liu, G. Kioseoglou, and A. Petrou, Kinetic and transport modeling of the metallorganic chemical vapor deposition of InP from trimethylindium. J. Electrochem. Soc. 142, 2086-2094 (1995).

[34] Theodoropolou, A., R.A. Adomaitis, and E. Zafiriou, Model reduction for optimization of rapid thermal chemical vapor deposition systems, IEEE Trans. Semiconductor Manuf. 11, 85-98 (1998).

[35] Warnick, S.C., and M.A. Dahleh, Feedback control of MOCVD growth of submicron compound semiconductor films, IEEE Trans. Control Systems Tech. 6, 62-71 (1998).

[36] Bayazitoglu, Y., M.N. Ozisik, Elements of Mass Heat Transfer, (McGraw Hill, NY, 1988).

[37] Zhou, J.J., Y. Li, D. Pacheco, H.P. Lee, and X. Liu, Virtual control simulator for closed-loop epitaxial growth, J. Crystal Growth 175/176, 52-60 (1997). 